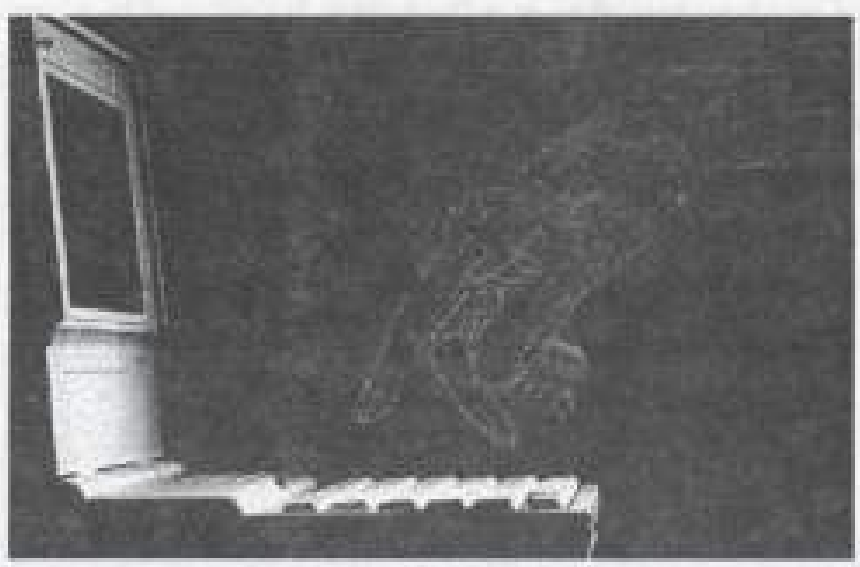

Maria Cecilia Sánchez Bravo*

\title{
ATroducción
}

Las personas, a trayes de lon tiempos, han setitides ta hecesidad de conservar ls informacion rocolectaita de diversas finmas, esta necesilad bumans se materializé en las biblietecss, palabra proveniente de]

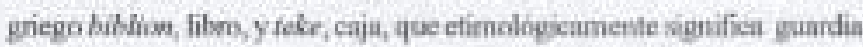
e custodis de fibros.

Para hablar del libeo digieal, es necesano recordar to histuria del liboo tradicional impreso en papel, porque su secoologia se haso en los principios $y$ fundamentow do este altimo.

Gutemberg al inwentar la imprenta en I440 dio inicjo a un nuevo desarrolla tecnológieo que nos ha permitide comocer la evolucion de la eicacia y del pensamiento de la humanidad. El papol, materia prima para la elaborsciobn de los lioros, evolucionó paralelamente con el desarrolle tesnológico de la imprenta. Desde la simple prensa de madera con sus tipos móviles que emplob́ Gutemberg para imprimir su priater libro, La Biblia, hasts ln mas sofisticada màquina de impresión de nuestros dias, todas ellas sin excepoibn utilizan of papel.

E.l fibto digital nació cano fruta de larges años de observación cn el campo de las artes gráficis y la informática. A princapios de 1993 se insicio el desarnolle del libro digital, usando is pantalla del computador

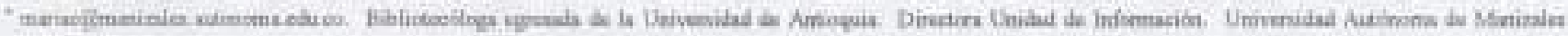


para rexmplazar el papel y erear una noeva teenologia basada en ci no papel. Klemath (1996, p. 19)

Los adeiantos realizados en cuanto a téeniès modernas de comunicación, bacen posible compartir grandes cantidades de información a pras velocidad entre los diferentes continestes y a bajos costos, io cial ha proporcionado la posibitidud de aprovechar dichos avances ténioas en el contexto de las biblioteeas, puesto que aụn prevulece la necesidad de hacer llegar el conocimiento en cllas conteribo a una población mayor, $y$ las bibibotecas actuales no pucden cabrir todas las necesidades de información de la comunidad.

Actualmente ta umsen forma de lograr que el oonocimiento repartide alrededor del mundo esté a disposición de todes, es la red de computadoren Internet, a truvés de la eual, pasando el libro a un medio dipital, se puede concentrar tods la información y hacerla asequible a cualquier perrona con aceeso a la red. 4.

La historia de recuperación de información incluye el desplazamicato del uso del papel, hacia los medios digitales. Las teorias acerca de in organización y el aceeso a la información, se han convertido en teorias del fiseño de bases de datos.

Seguin to plantea Arias (1995, p. 2) is edvesción y la comatrieación son las medios más seguros para generar signifieativos cambios en la sociedad: la tecnologia ha creado para ellos, ambientes que le permiten interachaar, transportar y difundir el cooceimiento a los tagares mas recóndilios. Ael planets.

In este documente se ba queride reflecionar sobee d apoyo que ofrece la bibhotesa a la edueación virtual, por consideraslo un asunto que mereces especial stencion. Internet ha introducido el onncepto de globalización en la economia y ya $s e$ ha excendido a fodos los sectores incluyendo el educativo, se han superado las harreras de tieitapo y espacio $y$ th vecnologia ofroce la posibilidad de tener la información disporvible como respaldo a cualquier proceso educativo, en el átio y a la hona que we necesite, Clano que, para poder disfrutar de los servicios de una biblictoca digital, es necessario desarrollar una infrestructura para garantisat conectividad permanente $y$ asegurar od acoeso a la red. comstituytodose en una limitante para los paises en ví de desarrollo, ya que requiere de altos presupuestos parn invertir en eguipos y en espacitacibin del talento humaner, estos paixes deben anter dar solueion a las necesidades primarias, como son Is alimentación y is aslud. La educteión se be convertide en articulo de primern neoesidad, pero aunque alguién halls dicho muy sabiamente "cl puctilo con hambre no aprende". ISerá entonces, que la toenologia definitivansente si esth incrementando is brecha estre los que tiencen aceeso y los que ao lo tiesen? La refiexión quodn sobre el tapele. Fste trabajo estín concebido a partir de la base quo se biene ssegurado el acceso a la red.

Al interior del documente se han utilizado los térninss: Bihliotecia Digital, Bablioteca Virtual, Bibinoteca Electrónica a Biblioteca del Futuro, a pesar de no ser plenameote sanómimos y que en la litenatura especializada se eneuentra gras cantidad y variedad de definicioness. pero en el modio se estín tatilisando indistintamente para derignar el conjunto de servicios de información ofrecidos a través de las TIC. Teenolegias de Informacton y Comunicación, para acerear al usuario a la información requerida, independienternente del sito y el formato en que se encuentre.

\section{CAMBIO DE PARADIGMA}

La Educación ha permanecido por muchas dboadas estacionada e inmodifienble, el doceate ha sido amo y sethor del aula de clase, siempte portador de la verdad y sudministrador def conocimientox, puede decirse que todo ef proceso ensel̂anza aprendizaje ha girado en torno al docente.

Pero se esth dando un yoan viraje en el proceso educativo y estia surgiendo auevas posibilidades parn ksdosc, el aprendiz quiere descubrir nuevos cumines para liegar a la verdad, existen nocvos medios para obtener conocimientos, la explosión informativa ha invadido todos Ios rincones del planeta y la tecnologia también ha tocado las pucrtas de la Edusaction.

E cambio no es una posibilidad para las instituciones de educación superior, es tha necesidad. Los estudiantes han eambiado, sus nocesidades han cambiado, la socicdsd ha cambiado, los negocios han cambiado, ef gobiemo hs cambiado, asi como ha cambiado el discurri de la vida nacional. (Rowley, 1998)

Se vive una ena de cambio ecentimuo y se entin viviendo los eonceptos de

Globalización, Diversidad, Apertura

Economica, 
Interdisciplisariednd to eual requiere una accptasción infetculhural, donde todos los seres humanos poseen valer. Lo importante es definir edmo las instituciones educativas van a satisfacer las necesidades de la sociodad on los proximos anios.

El mundo se esti moviendo boy hacia ta masificación de las modernas tecnologias informáticas y la superautopista de infornación, Ello ha generado grandes eanbios con respecto a la función de las bibbiotecas y el concepto do información. Las boblioteeas fienen usi gran responsabilidad no solo de ampliar $y$ facilitar el acceso al conocimiento rompiendo las barreras geografieas sino también de orientación a sus asuarios con respeeto a la multiphacación de fuentes y servicios de información que ha generado la implementacion de redes mundiales.

E rew que se peesenta hoy a las bibliolecas universitarias del mundo, es actualizarse en tecoologias de manejo de informacion tales como el hipertexto, la multimedia y las felecomunicaciones para tacilitar la búsqueda y recuperación de informscićn a los usuarios, infegrande los difereates formatos en que ella se encuentre, proporcionar eficientes servicios, de masiera que el usuario pueda concerer y obtener información mas alla de las fuentes locates y difundir a nivel nacional e infernacional el sonocimiento generado en et pais Esto permitira que las biblioteens establezcan verdadetos mecanismos de cooperaciòn e intercambio que redundarán cn una muximizacsön de los recursos, servicios apropisdes a las nucvas necesidades de los gsuarios $y$ ana amplia difusión del conocimiento, contribuyendo de esta manera a generar mayor desarrollo cientifico, vecnalegieo y sociseconomico.
Actualmente se está Jisfrutando, fas bondades de Internet, red que une gran cantidad de computaderes en el mundo y permito no salor el acceso a la información entre ellos, sino el traslado de ha infoemación đe un sitio a otro, independientemente de las distancias, los horarios, loa climas, los ígomas muestra de esto es ef servicio de carreo electrónico que agiliza dia a dia millares de eventos, transactiones e investigaciones en el mindo.

La comunidad académicn de EE UU sinthó ln nesesidad de eatablecer una red de las carueteristions de Internet, pers de uso exclusivo pan la acadeinis y la invertegación y como respuesta ha surgido Internet 2, red do alta velocidad, que constituye uma gran opeión de desarrollo para todos, ya que permite el traslado de información en ses diferentes presentuciones (voz, imigen, datrs. sic.).

Entre las cambios más significutivos que deberin efeetuar las instiniciones edusativas que descen set competitivas en el futuro, en felación a las necesidades de edocacién de adultos, se encuentra establecer la educación $\sin$ muros, de tal modo que los aprendices no tengan que desplazarse para obtener el cosocimiento, sino que pucdan haverlo desde su casa, st sitio de trabajo o lugar pecferido. Se utilizarh avarzada tectologia que permita libre fluje de información y eosocimiento. serí un aprendizaje individualizado e intersctivo. Tapisicott, D, \& Caston, A. (1995)

En el mundo contemporineo ln educación se constituye en la mayor posibilidad de dessrrollo social y humano. Es tan determinante el nivel edacativo de un pais en felsción eon sas oportunidades de sobtrovivit politica y coonćmicannenté en medio de an contexto de globalización, que la educación es un asunto prioritario del Estado y la sociedad eivil

Durante los proxinnes athos surviran muchas instituciones dedicadas especialmente a la cducación a distancia y parcece ser que la forma mis avanzada será el trahajo en red de los profesores y estudiantes sill una locafiraciòn geogrăfica común.

La enseîanza u distancia requicre hernamientas adicicenales y diferentes a las usadas por ef profesor en el salón de clase

Por otra parte, part cada indrvidae se propio nivel educativo determina en alto grado, las oportunidades de aceeder a los derechos bissicos progios de una sociedad demoerática y modernat el enpleo, la segurided social, th participación politica, el aceeso a servicios culturaies, a la cientia y a la kectnologia y el nivel de ingreso eonobimico eatan muy Eugados con las oportunidades que eada quien haya tenido de obtener mayores niveles de eduención. Por esto la educación se ubica en el rango de los dereckos fundamertales. Quzzá después del derecho a la vida, éste es el mís importante en tarto garantiza la posabilidad de desarrollar in vida.

En la sociedad de la información, et espacio y el tiempo no sont condieionantes de la interaccion social; un ejemplo de las nuevas formas de interacción ann las comunidades. virtuales: grupos de persuras que comparten un interes y que utilizan las redes informaticas como cantil de comunicaciōn harato y cómoda entre individuos espacialmente fispersos y temporalmente no stneronizados. 
La digitalización y los nuevos soportes electrónicos están dando lugar a nuevas formas de almacensar y presentar la información. las tutoriales multimedia, las bases de datos en linea, las bibliotecas electrónicas, los hipertextos, ete., on nuevas maneras de presentar y acoeder a la información; las cuales superan en cobertura las formas traficianales. Fs admimble como se estin transformando lis bibliotecas univeraitarias, de simgles depósitos de librea y revistas con salas de lectura anexas, están pasundo a ofrecer múltiples fuentes de infurmación electrónica.

La "utopia informativa" de la sociedad de la imformación es que tods la información esté al alcanoe de cualquiera, en cualquier momento y en easiquier lugar, acceder no sera el problema, el verdadeso problema de la sociedad de la información será la saturación y el ruido de todos los cansles, la difieultad de encontrar la infonmación que necesitamos, en esa masa gigantesea y en permanente crecimicato.

El papet de la excuela conno fuente primarin de información desapareció huve ya tiempo, perque la educación ee más que posecr informacion, ea tambicta conocimiento y sabiduria, hábitos y valores. Én el Libro Blanco sobre la Educación y la Farnación (Comisión Europen, 1995, p. 16), se afirma taxativamente que la sociedad del futuro serk una sociedad del cooocimiento y que, en dicha sociedad, "la cduckción y la formaciòn serian, más que nunca, los principales vectores de identifieación, pertenencia y promoción social"

En la sociobad del conocimiento, el aprendtaje no es ya una aotividud exclasiva del aula, vino que esth inmersa en todas las actividsdes sociales (trabajo, entretenimiento, vida hogerehill, elo,) y por tanto, en todos loe tempos en que dividimos nuostro die. La educación ya no se trata de una tarea infasti de preparsción pan la vids adulta y el trabejo, en realidaif es una parte cada ver más importante de auestras vidas.

Es importante difetenciar entre informaciỏn y conocimiento, of ecoocimiento implica información interiorizada y adecuadamente integrada en las estructuras eognitivas del sujeto, es algo personal $e$ intranaferible: no podemos tranamiter conocimientos, sólo infonmación, que puede, o no, ser convertida en conocimiento poe el receplor, de acuerdo a su estructura mental, a sus intereses y a sus conocinientos previok.

Las bibbotocas y la información en este presente y futuro cercano ofrecen al proceso educativo, a través de las redes de telecomunicaciones, las hases de datos y low hipermodion, la posibilidad de busear informasción quo responda a deseos y alraceiones muy personales; las bàquedas serda cada vez mas precisas de acuerdo a los intereses especificos de los alumnos que se estan educando, con profesores que cada vę mas tendran que planear ses ensefunzas para el grupo en su conjunto y al miseno tiempo gaiar los intereses y vocacioes personales, La biblioteca electranich oon Intemet y todos sus setricins sesan el complemente de la educaciôn general que da la eseuela; serí la pieza clave sin la cual no podràn funcionar los programas edueativos que pretendan estar insertos en li realidad social de su tiempo.

En el siglo XXI, más que munea se notará que el aprendiaje se da cen mayor intensidad y mas amplio espectro fueru del auls y el progratna oficial ofrecido por la escuela. La biblioteca electrónies, picza fundamental de la escuela y de la universidad a la par que el aula y el profesor, va a propiciar la interacción de fos estudiantos entre aula separadas no por un muro, sioo por océanos.

La tecnologia de la información ha redimensionado la biblioteca, pero también lo hará con las universidades y las escuelas ea general, pot lo qque los diseb̉os de estas tumbién variaran. Si hay caenbios al ofreeer la información y adquirir el conocimiento, tambièn deherá haber cambios al evaluar, por tun lado, a in bibliotecea cleetrónicar y los bibliotecólogos, que como ya se menciono va a reforzar sus responsabilidades educativas; y por otro lado, a la cscucla, a los profosores $y$ is los eatudiantes, que seguramente utilizaran diferentes caminos para adquirir su conocimiento y que sobre un plan de trahajo general, caifa uae de ellos profiandizará y recrearà aspeotos que rospondetan a intereses, actitudes y habilidales muy personales, por lo que habri diferentes formas de medir ha efectividaul, y la peoductividad.

\section{BIBLIOTECA DIGITAL}

\section{CONCEPTO}

Por BIALJOTECA se ha citendido el lugar en el que estain depowiadas diversas formas de información registrada. La acopción moderna del férmino base referencia a cualquicer fecoplación do información prescntada cn divornos formstos: mictofilms, revistan. Fbros, grabaciones, 
pelieulas dispositivas, eintas magnétieas $y$ de

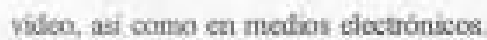

Para logma una biblinteca dipital, el material imprese es escancado en fomma ondensale, pars loceo ser importado a la base do datos donde. se indexa de acuerdo a parimetros ya establecidos para la posterior visualereación; el casoque la infiomación inicial ge encuentre ya en medio digital, èsta puede ser importads directamente a la base de datos. Posferiormente el vervidor permitira a los unuarios el acuess a los datos indexados aç̧ûn sean las caracteristicas o pormisos de aceeso definidos para eads uno de ellos La base def funciouamiento de una biblioteca digital es Intenset, ya que a través de la red se realizan todas las operaciones y servicios.

\section{OBJETIVO}

El objetion de la Biblioteca Digital es reuniz, publicar, conservar y difundir el mayor número posible de trabajos originales en formato digital y facilitar el aceseno a la mayor cencidad de usuarios

Se pretende que la Bibboleca Digital sea un putto de encusutro, un foro de intereambio de información $y$ experiencias y una opentunidad de publicacion abierta a todos lox -usararios.

\section{SERVICIOS}

Los servicios de infomación ofrecidos pot las bibliotecas digitales deberin ser flexibles, faseñados con base en teenologia, el sistema de información tendrá que tomar desde su đaserios inicial las demandas de los usuarios. Se trabaja en sistemas abiertes interconectables, Open Systems
Intereonection (OSI), que permitan ias tranafercneias de información y de atchivos.

La biblioteca del futuro enfiatirara su función de aycula al usuario, a fin de que a destancia y por ai solo acoodu a in información deseada. Parn lograr esto habra servicios directos e indiroctos, que sin haserse presentes estain ahi respaldando tudss las posibilidades que la bibliotess, con apoyos elevtrúnicos, ofreve para hacer uso de la informaciàn. En esta biblioteca se conservarán coleceiones y servicios basades en los impresos que eosvivirán con las colecoviones y servicios apoyades en los medics electrónicos.

Tudas los servicios que se afrezican en estas bebliotocas $y$ se deseñen en el futuro tendrum como meta principal acerear a los seres humanos, llamados hasta hoy usuarios, con la infocmaç̉os, ya sea acadennica, comunitaris, sociel, recreativa. Eteoneepto de los servicios se mantendrí; sin embargo, los alcandes y las modalidades se ampliarán en función del woporte en que se registre la información y de la disponibilidad teenológiea en cuanto equipo, eoleceiones y el aceeso a las telecomunicasiones.

Ya han surgidoy seguirin sugiendo servicies intimamente ligados a la tecnologia electronica y a las biblotocas de este tipo onme son los eomprendidos en el uso de interfaces grificse de usuarios, conocidos comb GUI, uadjo, animación, recursed multimedia, dispesitivos y herramientus sutoconfigurables.

Los ervicion ya establecidos y que están en vias de popularizarse sen:

\section{CONSULTA DELCATALOGOEN LINEA}

Las uauarios pueden aceeder a travós de
Internet, $\mathrm{n}$ ln información acerea de los lextes que alberga tisicamente in biblotesa.

\section{CONSULTA DE LIBROS DIGITALES}

Se pooden loer directasnente en el monitor del camputador los libros que han sido digitalizados

\section{ACCBSO A REVISTAS Y PERIÓDICOS}

Se ofrecen hipervineules a revistas y periódicos electrónicos, nacionales $e$ internscionales.

\section{INFORMACIÓN SOBRE EVENTOS}

Se informa sobre congresos, conferencins y reuniones en distintas drans deł conocimiento

\section{INFORMACIÓN POR E-MAIL}

De noterdo a us perfil de usarios previamente realizado, șe envin infícemaciồn via correo clectrónico. Además, los usuarios que ne Ioealicen la información podràn efectuar uns consultas a las Habliotecas a traves de eoereo electrónico y por este medio podrin obtener orientacièn o la información requerida.

\section{CONSULTA DE INFORMACION RE- GIONAL.}

Da aceeso a traves de Internet, a la información produeida ea cada regián y muy especificamente en las universidadea y centros de investigación eapecializados.

\section{CONSUlta dE BASES DE DATOS INTERNACIONALES}

Las Bases de datos en CD-ROM y en linea, facilitari a estudiantes, docentes, investigadores, empresarios. y a la comunidad en Heneral, localizat información relevante a aivel munual on todas las dreas del conecimiento, ya que 
Ins remitirh a los fextos completos de los articulos en las revistas especializadas.

\section{SERVICIODE ALERTA}

Segun ia especialidad de In Biblioteca, se colocarin para oonsulta a Iravés de Lnternet las tablas de contenido de las reviatas internacionales suscritas en linea. $y$ desde la tabla de eontenido se puede hacer ef enlace a la reviste electrobinicn.

\section{FOROS DE DISCUSION}

Las oumuninfoles acsdémicas tendran la oportunidad de sompartir de maneth asincoúnica, sus inquictudes $\mathrm{e}$ investigacianes.

\section{OTROS SERVICIOS}

- Videoconferenvia (oonversación vidootelefónica entro varios interlocutores simultàneamente, en la que pueden verse y mostrafse documentos

- Multamedia (traniferencia de información catre la computadom o ted y el ser humano a través de imágenes fijas y on movimiento, datog, woz y sonido en us solo sistema)

- Distribación de información vis celular o teléfonos mòvil.

Le Bibloteca del Futuro es una biblioteca que va a centrar màs que aunea su razón de ser en acerear la información a los. usuarios no importando la distanoia que exista entre uno y otro.

Para poder identificar la información adecuada para una necesidad especifica será indisponseble acercarse a toda la colección y navegar por varias de las rutas que ofreeen las rodes de telecoenuniesción e informacion

\section{VENTA.JAS}

- Los usurios pueden acceder a la información desde eualquier parte $y$ a eualquier hera.

- Hay una considerable reduccién de burocracia a través del acceso es linea a la informacion.

- No es neceirario el conocimiento de la estructura de un catilogo.
- Ia información no se erscuentm necesiriamente en el mismo lupar.

- El hipertexto ofree muiltiples posibilidades.

\section{CONCLUSIÓN}

Camhiar un paradigma es algo bien difical y que exige mucho earieter en quien se atreve a hacerlo, mas diffeil aûn en el ampo de la Fducación, donde los esquernas han permanecido intactos a través del tiempo. Peno dehemos atrevernos a concebir uma idea nuevi, con vida propia, que nos permita maximizar el desenupeño del talento humano y sacar provecho de la teenologia que tenemes a nuestro slannce.

del fenćmeno de la globalicación ha generado en el mundo una bopica de In competitividad que se intents introducir en los sistemas educacicrales. En este contexto, para que un pais pueda dcaurrollarse con exito en estas condiciones, es necesaro que tenga acceso a la información mis avanzada, al conocimiento cientifico y a las ixnovaciones fecnológicass Pulfer, D., Cänovas, L y de Pujadas, Q. (1999)

La automatración del sistema de teleoomumicaciones y ef increanento en la transmisión de dabos, como los peogresos besados en fibra óptica, has favcrecido la creación de una red de comanicación mumbial capez de transmitir grandes cantidades de informscsón mulamadin a todo el muodo.

La tecrologia ha ceasionado cambios sustanciales en la educacoots y ha generado gran variedad de modelos efucativos partiendo de la combinación de dos variablest tiempo y empacio, algunos de ellos están eapturando cada día màs personas, y en cambe olros han generado poltamicas donde se expresat sus oportultidades y amenazas, es apenas obvio que la comunidad edueativa e investigativa, reflexione sobre el cambio que se està viviendo.

La sociedad de la infarmación ha afeetado la función de las bibliotecas y de los profesionales de la información, quieries necesitan teplantear el papel que van a desempentar en la era digital. Los servicios bibliotecarios deben set modemizados y adaptados a las nuevas teenologias desarrollodas. De ahora en adeiaste, el estatus de una bibliotecz dependerá de la calidad de sus servicioss, mas que do la infraestructura del edificio o del tanaño de sus colecciones, sin perdet de vista que su razón de ser es conservar la enformación y transmitirla 
de una generación il otra, apoyando los procesos edueativos. independienternente del formato en que estos sean desarrollados

\section{BIBLIOGRAFIA}

Arias, J. (1995). Texnologias y educación, las rutas det nueve siglo.

Revista Interamericana de Nuevas Tecnolooias de la Isfornacion 1 (1): p.2

Brott, G, H (1995). Herramientas para la recuperacion de la informaciön en redes en el ambicnte universitarive hacia una biblioteca eibernética. Revista Internmericsna de Nacvas Tecologian de Información. I (1), 4-14

Cakva Gonvaler, J.J. (1999) . Et comportamiento en la bisqgaeda de infomación de los investigadores del area de. Humanidudes y Cesncias Sociales Disponible on:

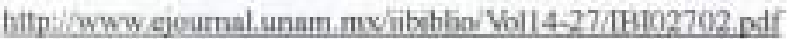

Card, f], (2000). Development and dissemination of an electronic bibrary of secial science data. Scoial Science Computer Review. $18(1), 82-86$
Cline, N. M. (2000) Virtual Continuity the chalkenge for research tharuries today, Educause Revicw, 35 (3), 22-28

Haddouti, H. (2000) Algunos aspecios de las biblsotocas en la en digital. Revista Interamericutia de Nuevas Tecologins de Infurmación 5 (I), 32.39

Jones, S. (ed.) (1999) Daine Internet Resecurch: Critical Issuks and Methods for Examinine the Net. Thousand Oaks, CA, EB.UU: Sage

Kkmath, Z. (1996). El tibro digital. Sistemas, Bogutk: ACIS. 19-23

Pulfer, D, Canovas, L. y de Pujadas, G. (1999). IX.Conferenes Theroanericana de Educscicin, Cula: OE!

Rowloy, D J, I ujan, H. D. and Dolence, M (1998), Stratege Choises? for the Aendermy San Franeiseo: Jossey Base. ISEN, 0-7879. $4067-4$

Tapseott, D, \& Caston, A (1995). Cambin de paradignas empresariales. Bogoth, Colombia: MeGraw-Hill 